Isadora Luana Flores ${ }^{1 *}$ Natanael de Alencar Samarcos Mahon ${ }^{2}$ Thiago de Oliveira Gamba ${ }^{3}$ Afonso Celso Assis ${ }^{2}$ Ana Paula Neutzling Gomes ${ }^{4}$ Sérgio Lúcio Pereira de Castro Lopes ${ }^{5}$
${ }^{1}$ Universidade Federal de Juiz de Fora, Campus Governador Valadares.

${ }^{2}$ São Leopoldo Mandic, Campinas.

${ }^{3}$ Universidade de Caxias do Sul.

${ }^{4}$ Universidade Federal de Pelotas.

${ }^{5}$ Universidade Estadual Paulista.

Correspondence to:

Universidade Federal de Juiz de Fora, Campus Governador Valadares.

Rua Gonçalves Chaves - 457, Pelotas, RS, País Brazil

E-mail: isadoraluanaflores@gmail.com

Article received on February 5, 2017. Article accepted on March 21, 2017.

DOI: $10.5935 / 2525-5711.20170002$

\section{Supernumerary bilateral tooth germs mimicking radiolucent bone pathology}

\section{Abstract:}

The immature tissues of teeth germs may mimicking odontogenic and non-odontogenic lesions. About this, an asymptomatic bilateral well-circumscribed radiolucency found during the routine evaluation is considered an uncommon finding and both supernumerary and maxillomandibular bone pathologies should be listed. Quite often, it is one clinical exercise to suggest diagnostic hypotheses to unspecific radiolucency image since cysts, neoplasms and dental anomalies are possible. Here, we reported a case of bilateral supernumerary teeth germs in a 12-years-old Caucasian patient highly suspicious of bone pathology due to completely radiolucent aspect. So, this is the first article to presents a case of bilateral teeth germs as an alert to early stage of tooth crypts in the differential diagnosis of mandibular circumscribed radiolucencies.

Keywords: Tomography, X-Ray Computed; Tooth, Supernumerary; Tooth Germ. 


\section{INTRODUCTION}

The presence of well-defined asymptomatic radiolucency in the mandible may indicate a diversity of bone pathologies, but bilaterality is a feature that also suggests an anatomical landmark or a variation of normality ${ }^{1}$. Among the odontogenic lesions, the lateral periodontal cyst (LPC) is an uncommon, but well-recognized developmental cyst, which commonly affects the adjacent areas to the roots of vital premolars ${ }^{2}$. The keratocyst odontogenic tumor (KOT), adenomatoid odontogenic tumor (AOT) and unicystic ameloblastoma (UA) are odontogenic tumors with variety of behavior and anatomic site of occurrence being that innocuous lesion can develops in the lateral radicular areas of premolars $^{3,4}$. Additionally, a non-aggressive central giant cell lesion (CGCL) also involves the interradicular areas and the corticalized radiopaque circumscribed contour can be observed such as seen in the odontogenic lesions $^{2-5}$.

Finally, supernumerary teeth are more frequent in permanent dentition and a single supernumerary tooth is a common finding in the anterior maxilla (mesiodens) and superior molar region. For the other hand, multiple supernumerary teeth occur frequently in mandibular premolar region ${ }^{1,6}$. The radiolucent aspect represents the early formation and it is not easily observed in this stage through imaging studies ${ }^{6}$. Moreover, the mandible is the most common anatomical site and isolated or multiple supernumerary elements can causing teeth reabsorption and/or represent a barrier to orthodontic treatment ${ }^{7,8}$.

So, the presence of supernumerary tooth can be considered and the dental clinicians should include this condition in theirs diagnostic hypothesis ${ }^{1,6-9}$. Simultaneously, the development of bilateral supernumerary mandibular premolars is a still more unexpected event, especially if found in the early stage. Facing that, we reported a rare case of bilateral supernumerary teeth germs mimicking typical radiolucent bone lesions. Interestingly, no previous articles were published in the English-Literature involving supernumerary teeth germs and radiolucent aspects.

\section{CASE STUDY}

A 12-years-old Caucasian male patient was driven by his parents to a private dental clinic for routine appointment and a periapical radiographic examination was performed. In this radiograph, a circumscribed unilocular radiolucent lesion with radiopaque halo was observed between the right lower second premolar and right lower first molar. No teeth and other bone alterations were identified (Figure 1).

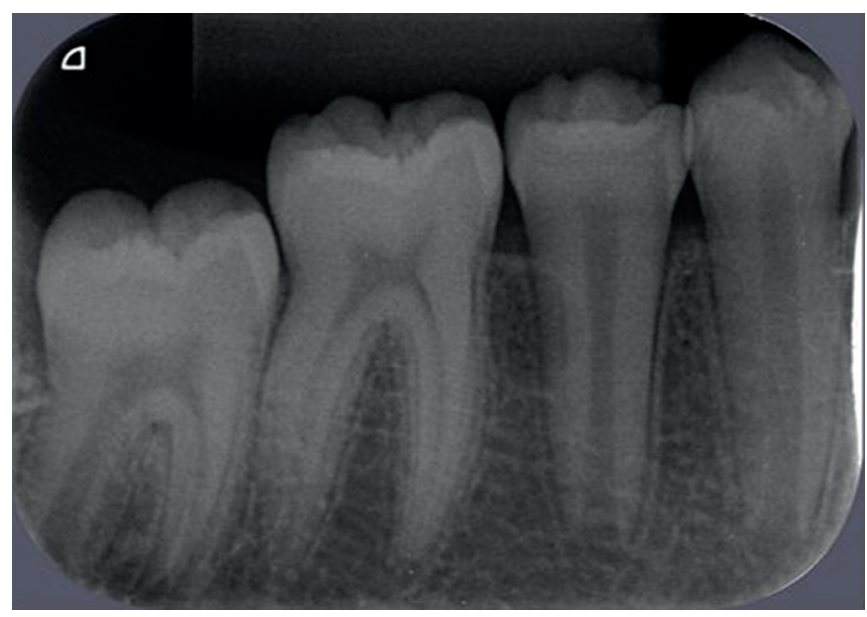

Figure 1. Periapical radiograph showing the well-circumscribed radiolucency between the right lower second premolar and the right lower first molar. Note the lesion superposition in the premolar root and maintenance of periodontal ligament space.

The medical history was not contributory. Moreover, due to the absence of clinical signs and symptoms and the radiographical aspects, odontogenic and non-odontogenic lesions such as LPC, KOT, AOT, UA and CGCL were included as differential diagnosis. An additional cone beam computed tomography (CBCT) was requested to establish the vestibule-lingual position, the relation of lesion with teeth, lamina dura maintenance and contribute to surgical planning.

The diameter of the FOV was fixed; however, a smallest height was selected to reduce the radiation dose. A well-defined oval hipodense area around 5,0mm of diameter related to lingual bone cortical and a thin sclerotic margin of lingual cortical was identified. Interestingly, a similar second image was found in the left mandibular side between the second premolar and first molar (Figures 2 and 3).

No absence of dental elements was observed. Based to images and pulp vitality, the clinical hipothesis of developmental odontogenic cysts was reinforced and a excisional biopsies were performed. The histopathological analysis revealed tissue fragments of tooth germs in bell stage with histo and morphodifferentiation composed by enamel organ and dental papilla (Figure 4). Thus, based to microscopic findings in association with a complete teeth counting, a final diagnosis of supernumerary teeth germs were reached. No complication were reported 


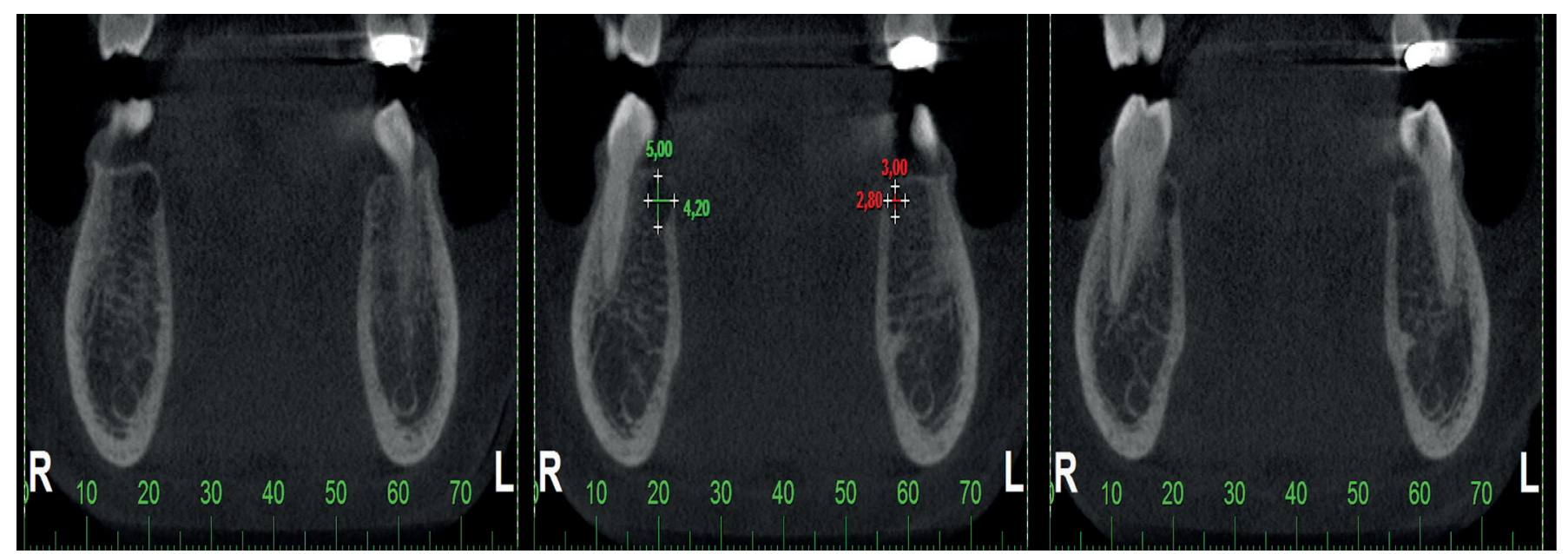

Figure 2. Coronal slices of CBCT highlighting the lingual position of the lesion, a mild resorption of mandibular right bone cortical and no bone displacement. A symmetric minor radiolucency in the left mandible was also observed.

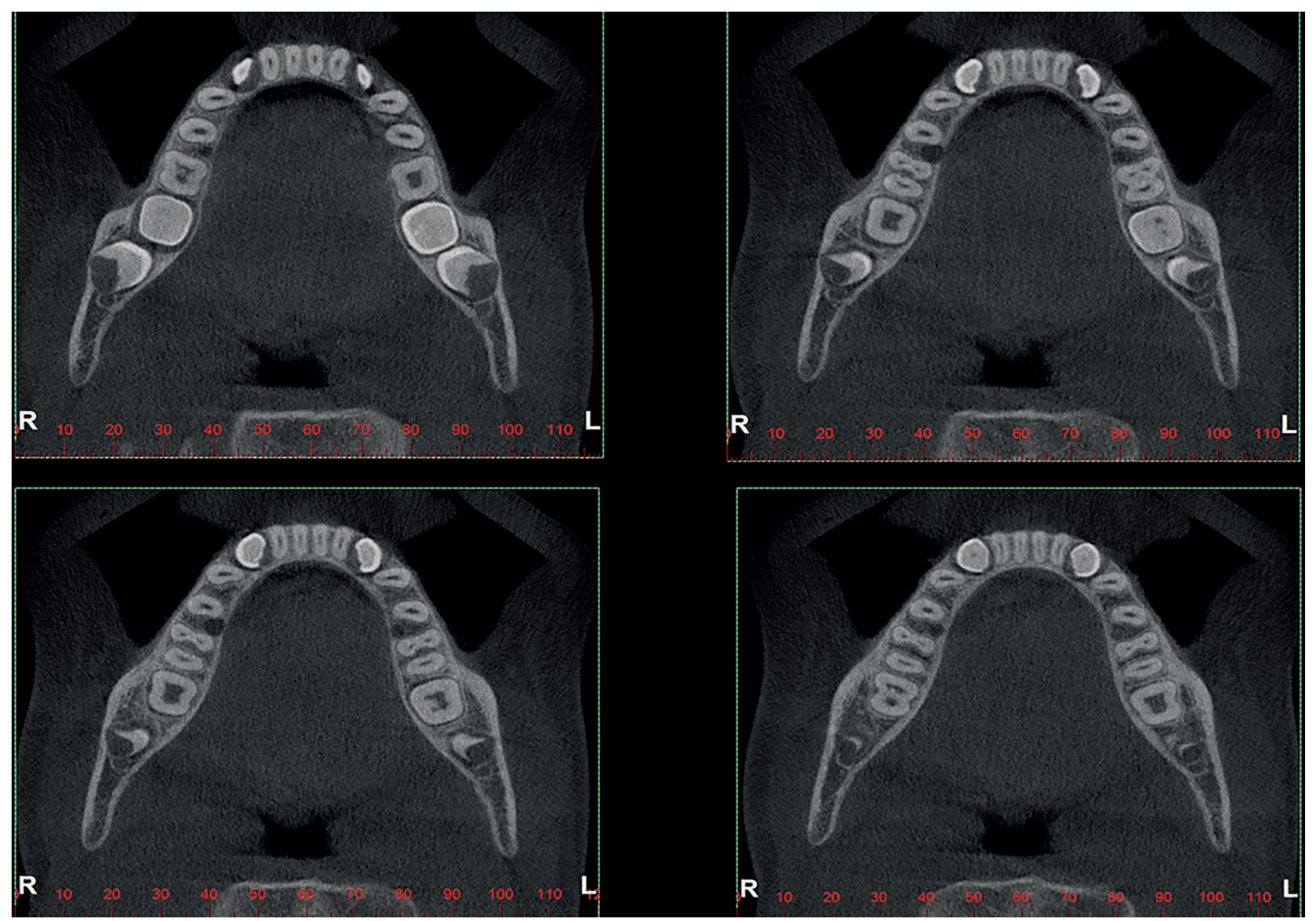

Figure 3. Axial slices evidencing the bilateral well-defined radiolucent lesions between the second premolar and first molar upon the mandibular cortical plate. 




Figure 4. Fragments of tooth germ in a bell stage composed by ameloblastic epithelium associated with stellate reticulum and intermediate strate besides loose ectomesenchyme with odontoblasts composing the dental papilla (H\&E staining, x40).

in the postoperative period; nevertheless, the patient discontinued the follow-up with his general dentist.

\section{DISCUSSION}

Supernumerary teeth are considered a developmental anomaly with no consensus about its etiology $y^{7,9}$. Nevertheless, the splitting of the tooth germ and/or hyperactive dental lamina are the main suggested theories ${ }^{7,10}$. These anomalies occur in beyond the normal dentition and are frequently observed incidentally after the intraoral radiographs during a routine dental appointments. Predeciduous, deciduous, mixed, permanent and postpermanent dentition can be involved with most frequent cases related with permanent teeth ${ }^{7,11}$. Furthermore, anterior maxillary and superior molar region are quite affected by single supernumerary tooth, while multiple supernumerary teeth occur commonly in the mandibular premolar $\operatorname{area}^{1,7,11,12}$.

The diagnosis of supernumerary tooth in the germ stage is an possible event in oral diagnosis. In the present case, supernumerary mandibular premolars were diagnosed in the postpermanent dentition and the hyperactivity of dental lamina is the highly suspicious etiopathogenesis ${ }^{7,13}$. In addition to this, Hedgehog, fibroblast growth factor, Wnt/ $\beta$-catenin and bone morphogenetic proteins families pathways have emerged as potential molecular pathways involving in the human supernumerary tooth formation ${ }^{13,14}$. Nevertheless, the exact underlying molecular mechanism is still unclear. In this context, the development of supernumerary premolars can be late and the formation occurs between 7 and 11 years after the normal dentition ${ }^{6,8}$. Moreover, supernumerary tooth germs commonly developing in the lingual position in relation to normal teeth; consequently, the imaging identification can be late even with their long time presence $e^{6,7,9}$. This event can be related with $\mathrm{x}$-ray conventional techniques which bidimensional aspects become evident and the anteroposterior view is impaired.

Based to the findings, panoramic and periapical radiographies can revealed unexpected diagnosis of supernumerary tooth germs ${ }^{7}$. Nevertheless, only clinical signs should guide the indication of $\mathrm{x}$-ray examinations in any dentition. Interestingly, the incidental finding of tooth germs was described in the present case during this age range in a very early step of tooth development. None previous article has described this atypical presentation of tooth germs in the English-Literature. Although the frequency of supernumerary premolars is around 8-9.1 percent and commonly they resemble normal teeth in shape and size, the radiolucent aspects is a rare event and should be included as differential diagnosis for maxillofacial bone pathologies ${ }^{7,15}$.

\section{CONFLICTS OF INTEREST}

The authors declare there are no conflicts of interest.

\section{REFERENCES}

1. White SC, Pharoah MJ. Oral radiology: principles and interpretation. $6^{\text {th }}$ ed. St. Louis: Mosby/Elsevier; 2009.

2. de Andrade M, Silva AP, de Moraes Ramos-Perez FM, SilvaSousa YT, da Cruz Perez DE. Lateral periodontal cyst: report of case and review of the literature. Oral Maxillofac Surg. 2012;16:83-7.

3. Goteti SH. Odontogenic Tumors: A Review of 675 Cases in Eastern Libya. Niger J Surg. 2016;22:37-40.

4. Sekerci AE, Nazlim S, Etoz M, Deniz K, Yasa Y. Odontogenic tumors: a collaborative study of 218 cases diagnosed over 12 years and comprehensive review of the literature. Med Oral Patol Oral Cir Bucal. 2015;20(1):e34-44.

5. Carlos R, Sedano HO. Intralesional corticosteroids as an alternative treatment for central giant cell granuloma. See comment in PubMed Commons belowOral Surg Oral Med Oral Pathol Oral Radiol Endod. 2002;93:161-6.

6. Langlais RP, Langland OE, Nortjé CJ. Diagnostic Imaging of the Jaws. $1^{\text {st }}$ ed. Baltimore: Williams \& Wilkins; 1995. p. 620-2.

7. Yassaei S, Goldani Moghadam M, Tabatabaei SM. Late developing supernumerary premolars: reports of two cases. Case Rep Dent. 2013;2013:969238.

8. Lara TS, Lancia M, da Silva Filho OG, Garib DG, Ozawa TO. Prevalence of mesiodens in orthodontic patients with deciduous and mixed dentition and its association with other dental anomalies. Dental Press J Orthod. 2013;18:93-9. 
9. Primosch RE. Anterior supernumerary teeth--assessment and surgical intervention in children. Pediatr Dent. 1981;3:204-15.

10. Liu JF. Characteristics of premaxillary supernumerary teeth: a survey of 112 cases. ASDC J Dent Child. 1995;62:262-5.

11. Parolia A, Kundabala M, Dahal M, Mohan M, Thomas MS. Management of supernumerary teeth. J Conserv Dent. 2011;14:221-4.

12. Scott JH, Symons NBB. Introduction to Dental Anomaly. $5^{\text {th }}$ ed. London: Churchill Livingstone; 1967.
13. Leco Berrocal MI, Martín Morales JF, Martínez González JM. An observational study of the frequency of supernumerary teeth in a population of 2000 patients. Med Oral Patol Oral Cir Bucal. 2007;12(2):E134-8.

14. Ge LH, Wang X. Recent advances in molecular mechanisms of supernumerary tooth formation. Beijing Da Xue Xue Bao. 2013; $45: 661-5$.

15. Brauer HU. Case report: non-syndromic multiple supernumerary teeth localized by cone beam computed tomography. Eur Arch Paediatr Dent. 2010;11:41-3. 